\title{
ENHANCING MOBILE COMMERCE: INSTANT MUSIC PURCHASING OVER THE AIR
}

\author{
J. FELIX HAMPE ${ }^{1}$ and GERHARD SCHWABE ${ }^{2}$ \\ ${ }^{1}$ University of Koblenz, Institute of IS Research, Germany \\ ${ }^{2}$ University of Zürich, Institute of Computer Science, Switzerland
}

Abstract: The advances of electronic commerce had an tremendous impact on the media industry already. In this paper we take a closer look on the audio industry and summarize major considerations found in the relating media theoretical literature. This leads to the main topic under study: the much more significant impact mobile commerce might have. We discuss present and future technology options, which could offer new distribution mechanisms as well as significant changes in the underlying value chains. By presenting an existing prototype development for ordering music via air we afterwards even extend the discussion to the consideration of seeing mobiles of third generation networks as a download and distribution devices. From the discussion of optional features of the prototype we return to the general discussion of a relevant subset of open research questions.

\section{INTRODUCTION}

The distribution and delivery of information, and especially that of music as a product has always been subject to significant changes due to technology shifts. Whereas broadcasting through licensed radio or TV stations seems well understood in terms of established models for fee collection, the invention of records, music cassettes or compact disc - digital audio (CD-DA) simply allowed charging via the physical media. But clearly, with MP3 coding of audio data (IIS, 2001) and approaches like Napster (Napster, 2001) or Gnutella (Gnutella, 2001) etc. those considerations of distribution, delivery and pricing have gained an overwhelming relevance.

The original version of this chapter was revised: The copyright line was incorrect. This has been corrected. The Erratum to this chapter is available at DOI: 10.1007/978-0-387-35692-1_36 
Internet based peer-to-peer services as used by Napster had been around for a long time (primarily in universities), but never before have we seen an approach for a very specific commercial domain with such a strong impact on its industry.

With a constantly growing user base of those systems for music distribution, there are significant technology implications when one thinks they all still require PC-equipment at the customer side, at least for the download of files. Surely it is possible to think of another distribution channel with nearly ubiquitous access, which can be connected to any PC?

So what are the implications if we look at music download services offered to an even larger user-base of the continuously growing mobile networks? As is well known, mobile e-markets show at least five major aspects of relevance (for an extended discussion we refer to (TIMElabs, 2000, pp 20):

- it is one of the fastest growing markets

- it offers ubiquitous, instant and fast access

- it will support mobile payment, thus allowing for instant settlement of transactions

- it promises around the clock service and thus continuous customer relations

- with IMT-2000 (UMTS) location based services could be implemented

With the emergence of the 2.5 or $3 \mathrm{G}$ mobile networks, an additional distribution channel for music (and later video, too) will be available, allowing for title selection and download, as well as accounting and billing over the air. This could be seen as a value added service, generating additional air time and specific revenue for mobile operators seeking to refinance their investments for spectrum licenses, both in terms of infrastructure and maintenance.

The question arises as to who will take over the role of (new) intermediary to set up this distribution channel. As will be argued in this paper, a variety of distribution channels will continue to co-exist, but the wider alternatives will allow radio stations to extend their business by offering instant shopping of music distributed via air, production and distribution of consumer customised audio products of any type and e-shop services offering all standard media products. By introducing some major building blocks of a much more general prototype, the paper provides a proof of concept with respect to the technical feasibility of this solution. CYS : Catch Your Song is a prototype based on 
Web and WAP technology which fulfils most of the requirements stated above.

This paper is based on a theory guided literature analysis and the development of a prototype as a prove of concept. Schwabe\&Krcmar (Schwabe/Krcmar 2000) argue based on Szyperski (Szyperski, 1971), Nunamaker (Nunamaker/Chen 1990) and the understanding of the German IS community (Heinzl et al. 2001) that designing sociotechnical innovations is an important task of Information Systems Research.

\section{RADIO STATIONS AND THE MUSIC CONTENT PROVIDERS}

In the following discussion we concentrate on the literature relating to the German music market, but our conclusions are equally valid for other European and overseas media markets.

\subsection{The current situation of the radio stations}

In Germany, competition in the radio business began about 15 years ago with the appearance of private commercial radio stations (Filk, 1996). Up to then, there were only public radio stations. Competition has forced public radio station marketing to the point where critics no longer see any significant difference from private radio. Furthermore the listener market has changed: with the increasing abundance of other media, radio has become a byproduct consumed during other activities (i.e. a 'stand-by-medium'), e.g. while driving a car. This has made it increasingly difficult to bind listeners to a certain program; and radio stations therefore prefer to try to bind listeners to their station. Typical components of modern radio marketing strategies are (see (Seidel/Libertus, 1993; Heinrich, 1994; Heinrich 1999; MühlBennighaus/Zerdick, 2000; Schumann/Hess, 2000)):

- The creation of a station identity that is transferred through jingles, and the structure of programs etc. The listener should feel "at home" with his radio station. Similarity of program structure makes orientation easier and reduces the listeners' tendency to switch to another radio-station.

- The building of a listeners community (see e.g. the community of SWR3 (SWR3, 2001)). This community receives extra benefits (e.g. reduced entrance fees to music events) and extra information (mostly over Websites), has the opportunity to communicate with the radio moderators 
and is involved in running the radio program. As most German radio stations are local or regional, listener communities have a 'natural' geographic basis. For the radio station, a strong listener community is a source of considerable commercial and political power (the latter is particularly important for public radio).

- the creation and marketing of events with visibility beyond radio. The transmission of live concerts has been a traditional task of radio stations. Modern radio stations go much further: They create their own music festivals (broadcasting only small fractions of them) and co-market tours of famous artists. They thus both create their own content and make their communities more attractive (for further information on community building ref (Kim, 2000)). During the 1990s new media and product alliances between radio stations and the music industry led to merchandising opportunities for both partners.

The latter two efforts have led to an increase in collaboration between the radio stations and their content providers. Both have become close content partners, each one owning a different distribution channel. The characteristics of the distribution channels are actually so different that radio and CDs are seen as two different products by the general public (for an extended treatment see (Zerdick et al., 1999; Goldhammer/Zerdick, 1999; Szyperski, 1999). An increasing number of radio stations have discovered the Internet as an additional broadcasting medium. While currently the traditional program is mainly broadcast, radio stations are already taking command of the market for future value added services. This potential will significantly increase once digital audio broadcast (DAB) is introduced more widely into the market (Digitalradio, 2001).

\subsection{The current situation of the content providers}

There is tremendous pressure on the music industry for change (Middelhoff, 2000; Marathe, 2001). Music is a non-physical product and can thus be delivered entirely over the Internet. Recent inventions in audio compression, increasing network bandwidth (see the discussions further below) and the lack of law enforcement have led to an explosion of - largely illegal - distribution of music content over the Internet (for a discussion see (Dernbach et al., 1998; Neverla, 1998; Ludes/Werner, 1997; Goldhammer/Zerdick, 1999)). Music listeners have not only learnt to like over-the-net distributed music because it is free, but also because it provides easier and more flexible access to a larger volume of music titles than can be 
found in conventional record shops. Music content providers ${ }^{1}$ have to find ways for online music marketing and reestablishing legal distribution. One approach is to make the internet "safer", i.e. to establish mechanisms on the net that allow content providers to collect royalties for their music. Technically, this requires a music-format that allows control over the copying of music titles, as well as avoiding illegal playback. The Secure Digital Music Initiative (SDMI 2001) has been formed with this objective, but has failed to produce tangible results. As the MP3 format is already very widely used, establishing a new standard that incorporates no further significant advantages for the consumer is at the very least an up-hill-battle, and more likely a hopeless task. Institutionally, "safe" music distribution over the Internet implies the build-up of legal electronic commerce sites for music distribution. As an example we refer to the "Music on Demand" platform of Deutsche Telekom (AOD, 2001). A prerequisite is a suitable business model for marketing music over the Internet. The recent takeover of Napster by Bertelsmann shows that music content providers are beginning to become more proactive in distributing their content over the Internet. Still, legal music distribution over the Internet may always stay problematic.

With respect to our topic of using mobile networks as distribution channels we note the following arguments which should attract content providers:

Mobile systems based on cell phones may become a much more attractive distribution channel:

1. The control of copyright in mobile networks could be realized more easily, because user authentication is performed by the subscriber identity module.

2. The customer base of mobile phones is already larger than that of Internet users and market penetration will increase in the foreseeable future (Durlacher, 1996).

3. Customers are already used to paying for mobile phone services (which is not the case for Internet services).

${ }^{1}$ For the purpose of this paper, the general term 'content provider' means all elements in the value chain of the music industry from the invention of the music piece by the artist to the production and multiplication of the music media, excluding the distribution and marketing. For a discussion of the value chain ref. later sections of this paper. 
Despite these obvious advantages it will not be technically and economically feasible and reasonable to distribute all music exclusively over mobile phone networks for some time to come. But diversification of distribution channels as in similar branches will be observable. Thus, any mobile service for music marketing will also rely on other media channels for the transfer of content. Here radio stations may be the ideal partners for content providers. The remainder of this paper will introduce a concept for such a partnership.

\subsection{Division of labor and value chain}

The fundamental requirement to form such partnerships is the adjustment of current value chains or the creation of new ones. For the justification of this statement it makes sense to analyse the current value chains. Roughly, the value chain up to now shows the following traditional players (see figure 1) according to the detailed discussion in (Zerdick et al., 1999) and especially (Kulle, 1998).

Artists create their work (e.g. a pop song) and originally hold the rights. The work is then (traditionally) published and/or performed in collaboration with (concert) organisers and publishers. The third step is the recording of the piece and the production of the master tape. The organisational and technical environment for this step is supplied by producers and studios. This is also the last step in which the artist is directly involved. Media factories afterwards multiply the audio media (e.g. a CD-DA) and publish it. The product is then sold by shops and clubs (i.e. primary exploitation). If the music titles are publicly preformed or radio stations broadcast them, license fees result in a second stream of revenue (i.e. secondary exploitation). The consumer then buys the pieces, listens to them and copies the content privately (within and beyond the legal limits). 


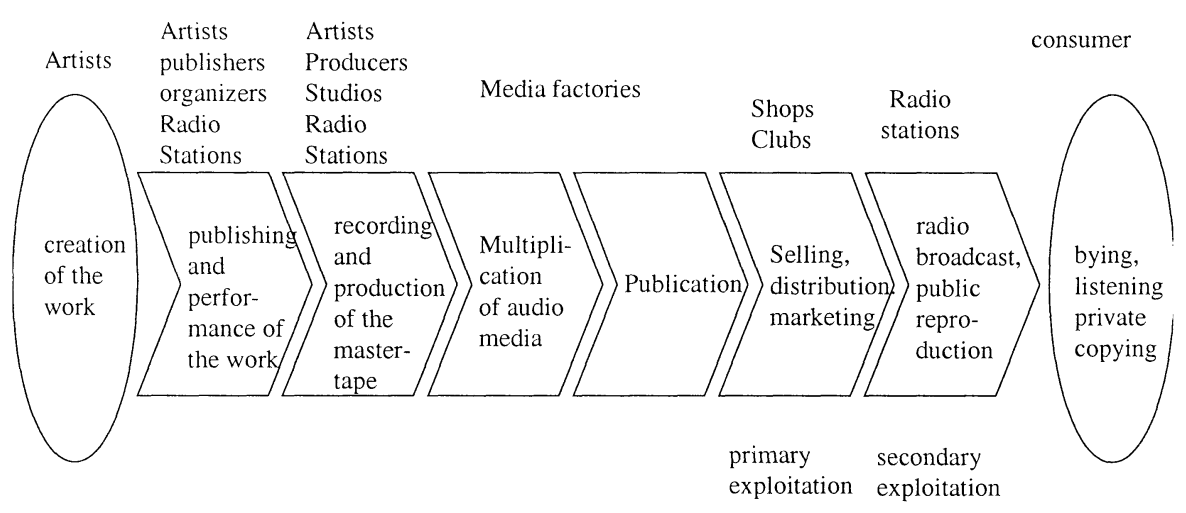

Figure 1. Value Chain of the Music Industry (based on (Kulle, 1998))

This value chain is heavily threatened by internet based electronic commerce: The whole value generation process may get cut off, if the consumers copy and distribute most of their music titles privately without paying royalties. The digital recording has provided a format that allows copying (almost) without loss of quality. The resulting private copying and selling of CD-DA has been the biggest source of loss for the music industry. An even much more significant threat is, however, seen in the illegal distribution of music over the Internet. Here, the music industry faces an illegal distribution channel for music titles that is far better organised than schoolyard CD-DA markets. In many senses an Internet-based value chain is also superior to the traditional value chain in terms of flexibility, availability, time to market and cost: traditional multiplication of audio media, publication and selling, distribution and marketing can be replaced by Internet-based value generation processes. For a sound and detailed discussion of the structural changes to the value chain through online-musicdistribution we refer to (Kulle, 1998).

These highly visible changes have moved other changes to the value chain into the background. One such important change of the last decade is 
the increasing involvement of radio stations into value generation prior to the secondary exploitation stage. Radio has traditionally been involved in the phase of performance of work, particularly in terms of classical music. These performances have also been recorded by (public) radio stations. Furthermore, radio stations are increasingly involved in the selling and distribution of music titles in three ways:

1. Radio stations broadcast commercial advertisements on new music media.

2. Content Providers and radio stations co-market events (concerts etc.) and implicitly the underlying music

3. Radio stations select and broadcast new music titles and thus have a decisive influence on the attention a music title or an artist gains.

Broadcasting of music content is less harmful to the economic value than broadcasting of video content by TV-stations, because music is a repetitive good: if one likes a song, one is inclined to listen to it over and over again. Even though a listener can copy the content from radio broadcast, the music industry has learnt to live with it because radio delivery had several important restrictions:

1. Quality: Radio delivery is typically of lower quality than digital originals; the widespread delivery of radio programs over cables has increased quality significantly. With the diffusion of digital audio broadcasting, quality will cease to be a problem.

2. Content selection: Radio is a push service without history and without future: The moderator selects a title and plays it; typically the listener does not know in advance when a certain title will be played and does not have an opportunity to access a played song afterwards (A minor exception to this is play lists published to communities in advance as an extra service to members only (SWR3, 2001).

3. Reimbursement: The content providers are reimbursed for their content by the radio companies through public intermediaries. However, reimbursement is low in Germany and a source of constant conflict between radio stations and content providers (Kulle, 1998, p. 276).

4. Legal Limitations: Radio programs typically play only selections of albums, but very rarely the total album.

The music industry and the radio stations have co-existed in a relative stability that was based on balanced market power as well as a symbiotic 
relationship to one another. The sources and critical aspects of this "equilibrium" are:

1. mutual interdependence: the vast majority of all music titles broadcast is owned by the music publishing houses, but no music title has a chance for commercial success if it is not broadcast by the radio stations.

2. control over the artists: Artist are typically controlled by the music industry (through contracts). This control is a major source of power for the music industry.

3. control over the listeners: Radio stations have instant access to the vast majority of all listeners and the ability to influence public opinion.

4. protection of the radio stations by law: The market for radio broadcasting is regulated by law. In Germany, these regulations entitle radio stations to broadcast content for a non-negotiable and low fee.

The most fundamental source for a stable and mutually accepted coexistence is, however, that both players target different listeners needs and thus effectively different markets. This stability may very well end soon as both players move into one another's markets. The music industry can and very likely will build up its own music-communities. It can offer for each community or even for each individual radio-like programs for a monthly flat rate. The radio stations can use their wide user basis and direct access for selling content. This latter opportunity will be focused on in the remainder of the paper. We will introduce concepts and a prototype for mobile commerce based on radio programs.

\section{THE POTENTIAL OF MOBILE SYSTEMS}

Mobile systems have technically diversified over the last few years by the introduction of new standards, e.g. BlueTooth, 802.11 (wireless LAN) as well as UMTS. They all show some similar features, like cellular structure, but in detail they differ significantly. The same holds for the primary type of usage, reach, bandwidth or cost. The field for study is so wide, that it certainly can't be treated in detail within this paper. We summarise therefore a few aspects relevant for the discussion of the prototype solution. 


\subsection{Technical potential}

The first issue is bandwidth. As is known from streaming audio, acceptable but low quality music playback can be achieved with common modem transfer rates (verifiable by using the Windows Media Player option 'Radio Tuner'). The basic idea here is buffering before playback, slightly higher connection speed than the audio transfer rate and sufficient processing power. But in downloading MP3 files which provide higher audio quality, more bandwidth has to be available. Compared to regular CDDA less storage is needed for the recording of music. MP3 allows for compression ratios of approximately $1 / 11$ if nearly CD-quality is required (bitrate of $128 \mathrm{kbs}$ ). But the resulting file size is still huge. An example may clarify this: the title from Mariah Carey's album labeled '\#1's' called 'When you Believe' has a playtime of 4'35', or $275 \mathrm{sec}$. Per second we would have to transfer 176400 byte $(44,1 \mathrm{kHz}$ sampling frequency and 16 bit per sample, stereo). With MPEG 1.0 layer 3 coding and a bit rate of $160 \mathrm{kbs}$ (for even superior quality) the resulting file size is $5.3 \mathrm{MB}$, whereas the original file size is $46.4 \mathrm{MB}$. For an extended technical treatment of MP3 coding and its successor AAC we refer to (MPEG, 2001) and (AMM, 2001).

The bandwidth offered today in GSM standard based mobile networks is $9.6 \mathrm{kbs}$ and will effectively increase to about $43 \mathrm{kbs}$ with GPRS only, but with EDGE or UMTS we will realistically approach $384 \mathrm{kbs}$ (Durlacher, 2001 , p. 13]. Whereas the former compares with the low quality music transmission via modem connections, the latter is sufficient for higher quality MP3 transmission. While listening to it the transferred data could be stored in the mobile device for further use.

The playback of MP3 files is no problem, as can be seen from the variety of mobile phones with onboard MP3 players available today. As an example we refer to the SIEMENS SL45 allowing for an optional memory extension of $128 \mathrm{MB}$ (Siemens, 2001). Thus we could have about 25 songs as above.

As has been evident over the last few years, memory capacity and size of handsets is no longer a critical issue. The technology found in other devices like digital cameras is offering micro disks with $1 \mathrm{~GB}$ and certainly we will see even higher capacities soon (IBM, 2001).

\subsection{Business Potential}

Mobile commerce per se has some interesting features which are worth considering briefly: 
- identification of users via SIM authorisation leads to regarding a mobile as a very personal piece of equipment and fosters the concept of non repudiation

- with respect to the stored directory information or other PDA type data the device incorporates personalised information to a large extent, thus lowering barriers to store personalised data on directly related web frontends

- in most contracts for mobile phone utilisation the payment for any service is coupled with direct debit facilities, thus converting the mobile to support instant payment to third parties

- by use of the CallerID one could realise a personalised versioning of any data stream prior to downloading, thus allowing for copy right protection or at least watermarking

- as location-based services become available, it will be interesting to see closely related business opportunities emerging, e.g. receiving audio data containing tourist information if the mobile is located close to a monument and the specific channel has been subscribed etc.

With those considerations in mind, areas of opportunity for mobile commerce have been seen in many areas, primarily in ticketing and ordering, mobile banking and payment as well as edutainment, see (TIMElabs, 2000) and (Durlacher, 2001). With respect to audio data we will concentrate here on instant ordering for later access via any other media channel or spontaneous download and listening.

Whether a pay-per-listen or pay-per-download approach will be followed depends on many factors. One is certainly the pricing scheme for content and for transfer time, while another might be the ease of use or access.

As was shown by the early disappointment of the Short Message Service, followed by its late but ongoing tremendous business success, the acceptance of new services is difficult to predict and seems not be related to established patterns found in other media. SMS not only offers an anachronistic user interface for sending very limited strings of max. 160 characters, but is also an extremely expensive communication environment compared to common prices on the internet.

For sending one SMS the average transfer charge is between $€ 0,08$ (network internal) and 0,20 for external messages within the German mobile networks. Those fixed prices ignore the effective length of the message. Assuming that one could really send the full 160 chars for $€ 0,20$, the extrapolation for transferring 1 MByte indicates an amount of more than $€$ 1.310 ,- which would be a staggering price in the IP-world. We do not consider that this extrapolation is really meaningful, but it clearly indicates 
that users of mobile networks are used to, and willing to, pay significantly higher prices for basic services compared to the internet. Furthermore, mobile users are used to paying for transfer and for content, as many of those SMS-based instant messaging services, e.g. stock market or specific news channels, as well as unified messaging services indicate.

\section{AN INSTANT SHOPPING APPROACH FOR MOBILE DEVICES: CATCH YOUR SONG}

\subsection{Business Idea}

Regular CD shopping, as found for example with amazon.com, can be set up by use of nearly any web-shop platform. Extending this service to allow order placement via mobile handsets is substantially more challenging, as the user interface design for the selection process of titles is non trivial. The idea of CYS does not tackle those general problems. But the approach allows the user to order any song whilst it is being played on the radio, without knowing any details such as title or artist etc.

There might be many situations where people listen to radio. Without any further empirical analysis (which could certainly be undertaken with currently available commercial media data), we can imagine that listening to the radio while driving in the car might be a typical occasion. As soon as a new song is released, most radio stations will introduce it to their audience, thus fulfilling their marketing contracts or simply by facing the competition of staying up-to-date compared with other stations. That is the very moment instantaneous buying might offer a competitive advantage. As the customer has subscribed to a community of one or more radio stations, s/he dials a specific number or URL via his/her mobile and connects to a dialogue which shows those titles played within the last minutes (e.g. the actually played title and the one before that). With one further selection and an accept click the ordering, the delivery and payment process will be executed. A variety of delivery forms are possible, i.e. direct download via air interface into the mobile which owns the feature of an MP3 player, or storage on a web host for later download are possible options. The authentication of the user has been performed prior to the connection setup via calling line identification. The billing process could be - as today in many call-by-call solutions handed over to the mobile carrier, which receives a call detail record from the service provider for any single transaction. The associated accounting 
data are kept by the service provider (i.e. the radio station) and could be used for further cross- and up-selling activities as well as marketing campaigns. Certainly, this will work only in a legal world with explicit user allowance for dealing with those data.

At any time the customers may access their personalised web-store and rearrange, delete or enlarge their title selection. They may order any form of delivery on any medium in any format at any time, or even may generate different title arrangements and send them as gifts or loyalty bonuses to friends or business partners. The radio station, obviously working with subcontractors at least for the logistics, will take over the role of traditional intermediaries via this e-business approach. Beyond the production phase of a master tape, record or digital audio stream, the complete later phases of the value chain could be influenced by the service provider.

So far we have concentrated our considerations exclusively on the radio stations, but it goes without saying that either Music-TV stations of today or any interactive TV-station of tomorrow could offer this service, too. The reason we have concentrated on music is, in fact, the bandwidth availability today and in the near future - but in principle the idea could be extended to, or beyond, any movie material.

An important consideration is the issue of dis-intermediation of traditional music shops on the one hand, and the re-intermediation by those service providers on the other hand. We argue that radio stations are well prepared and positioned to adopt this role. Revenue generation could be achieved by access and / or content fees, by sophisticated forms of advertising, by cross or up selling via all accessible media channels, or even by selling data collected from profiling (obeying the law of customer protection). For an extended treatment see, for example, (TIMElabs, 2000, pp. 155]. In the following section we describe the functionality of the mobile ordering component within CYS in more detail.

\subsection{Functionality of the prototype}

CYS (Catch-Your-Song) has two components:

1. A web-based component for the individual music archive and for the participation in a music community. This system can be used on any conventional computer connected to the Internet with a standard browser.

2. A mobile access component for "catching songs" that are currently on the air in selected radio stations. This mobile access system is currently 
based on a WAP-enabled mobile phone, but could also be integrated into a car-radio etc.

The system should be used in the following manner: interested radio listeners firstly register their radio stations and personal interests, using the web-based components, and subscribe as members of a music community. Whenever they hear a song on the radio, with a few clicks they can "catch" the song. We refer in the following description to the sequence of screen shots in figure 2.

Assuming they have selected SWR3 (first picture), they will see the current song in the cellphone interface and catch it with one selection (second picture and third picture). They can then either finish the transaction or continue processing the song (fourth picture): Either they order the single, order the album, download the song onto their cellphone or send an electronic greeting mail with the song attached. Finally, they decide on their preferred payment method. Once the users return to their desktop with Internet access, they will find a list of the radio stations and their programs, as well as an updated list containing their personal library of all songs caught. At this point they are once again offered all ordering options.

We now discuss the technical feasibility of this concept from the point of view of the service provider and discuss alternative options of the service concept.

Currently we have realized a CYS prototype, which simulates radio stations with a random selection of music titles from a database. We have not completely implemented the payment system, but simply generate a record entry in a payment database for each transaction as needed for accounting and billing processes. The remaining features of CYS including the interaction between WAP and the Internet has been implemented and tested. A major challenge has been the design of the interface allowing for minimal interaction on the mobile phones as well as sufficient ease of use for the web-system. 
Air

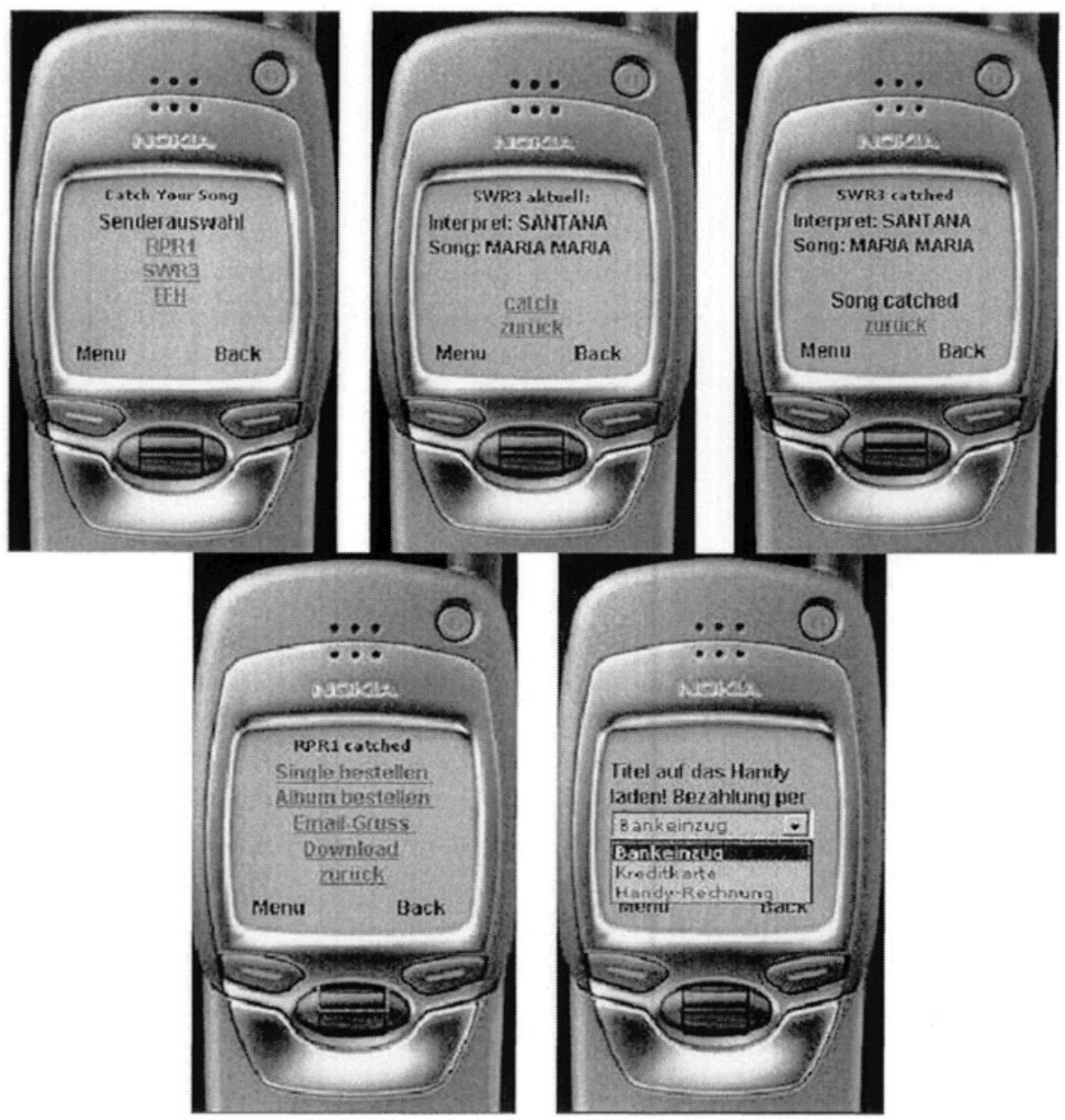

Figure 2. WAP-Interface CYS2 ${ }^{2}$

A real service provider would certainly face some more technical challenges:

${ }^{2}$ Screendumps are reproduced in low quality to reduce file size. A published version will have high-resolution screendumps. 
- The system should be scalable, allowing for heavy peak loads

- In a legal environment each delivery should be watermarked using the CallerID or any other suitable item for unique identification of this specific customer

- Transaction logging has to be performed for later claims etc.

- Considerations of insufficient connectivity (especially for transfer via air) have to be undertaken and formulated into contract clauses

- General security policies with respect to the web-shop operation have to be applied

Most interesting is the security issue once the mobile platform has been left, as it leads to the well known problems in general web-commerce requiring high level trust centre functionality.

Economically, the timing of the payment is crucial. On the one hand, payment issues should be hidden as much as possible. Settling payment can distract listeners from their current activity (e.g. driving a car). From this point of view, it would be preferable that the users initiate those transactions in non-mobile situations from their desktop. This approach, however, might have the following disadvantages:

- instant, emotional decisions are the dream of marketing. If the listener is just hearing the song and finally deciding on buying it, s/he is in a happy mood (because of having just listened to a song s/he obviously likes) and must decide on a relatively small amount of money (for one song). Once listeners have returned home, they might get second thoughts, particularly if they realise the summed cost for a larger number of songs.

- postponed buying is hardly possible, if there is large-scale illegal music distribution over the internet; listeners could collect the title information with catch-your-song and then get the songs from the Internet instead of buying them! 


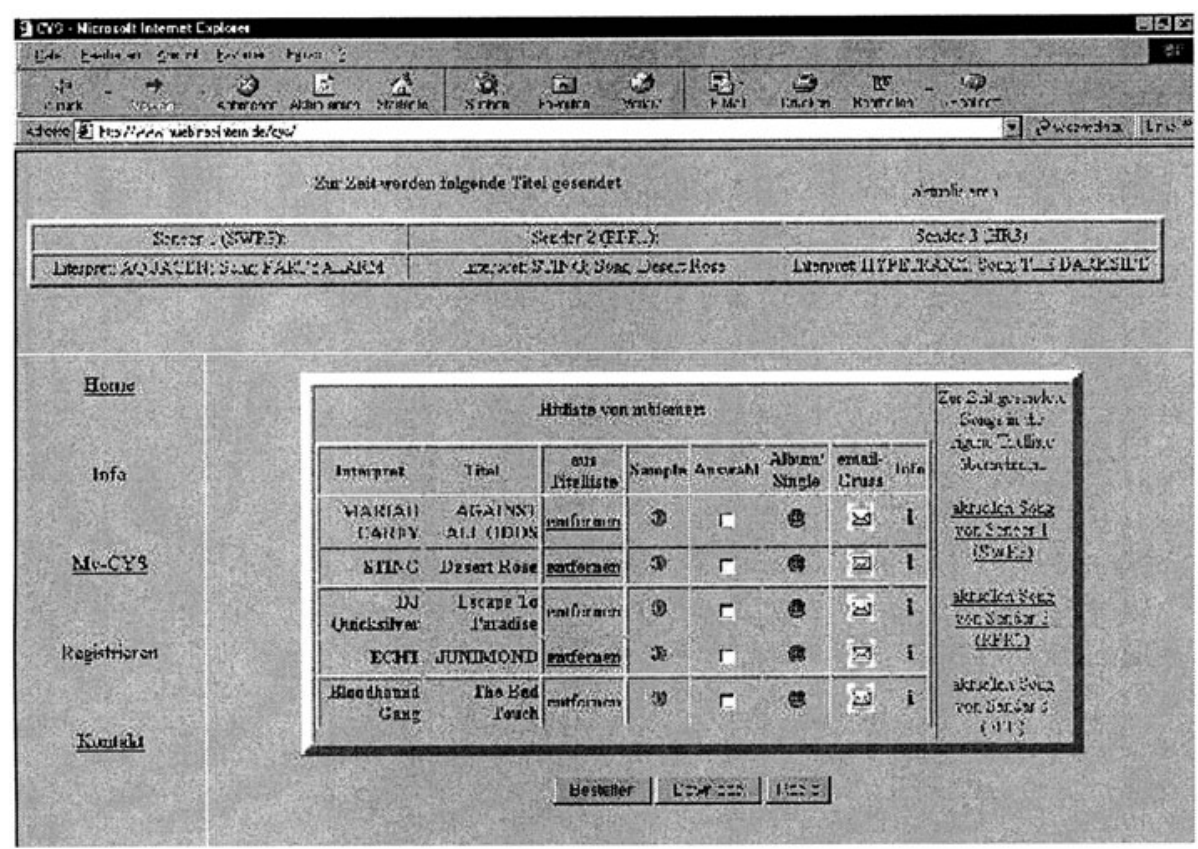

Figure 3. Internet Interface

Thus, ideally, catching a song and purchasing it is combined into one transaction performed by a very few clicks. In fact, the average length of the transfer process might induce some non trivial problems with respect to complete delivery, non repudiation of delivery etc.

CYS song is only the beginning of radio service diversification, the path to future features is sketched in figure 4.

A CYS-user cannot just listen to radio songs, but can also order the song using his/her WAP-mobile and access the selections over the Internet. We have shown that this can be implemented. The newest generation of mobiles will be full-featured MP3 players with infrared or BlueTooth connectivity to notebooks, desktop or hybrid multimedia devices. Thus, as soon as bandwidth becomes less expensive, songs can be downloaded to those mobiles or PDAs and listeners can carry their individual current best-ofselection in their pocket. With the option of transferring those downloads, the problem remains how to avoid illegal peer-to-peer distribution. But certainly the system design should allow that any data should be freely transferable by the owner and is not restricted for limited use with only one single device. 


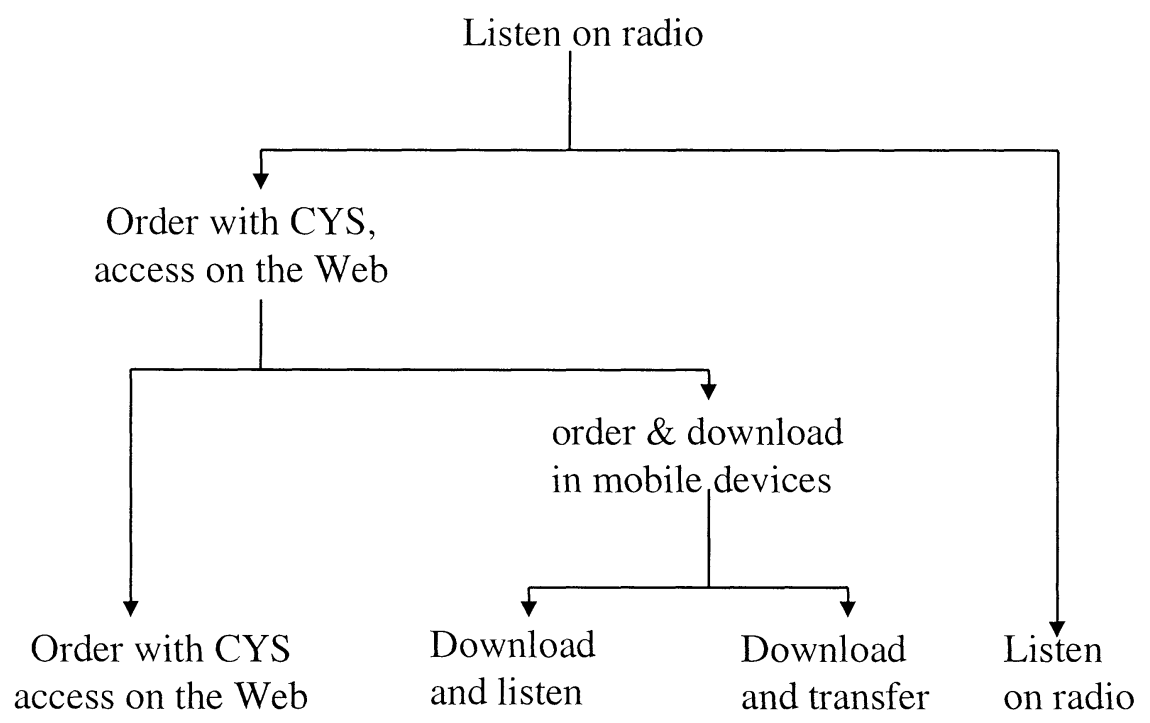

Figure 4. Service diversification

\subsection{Implications}

New business ideas like CYS open a new era of co-opetition between radio stations and the major media companies. Both have a lot to gain from cooperation but inevitably there will be an increasing overlap in their activities. The most fundamental issue will be: "who owns the customer"? One argument is that customer ownership comes with payment. This argument would imply that the music industry will very likely run CYS and any other advanced services, since they are in a much better position to build up a new payment system. The radio would just become one interface for digital music commerce among others. Another argument is that customer ownership comes with community ownership. This argument implies that the radio stations will run CYS, since they are in a much better position to create and support communities. While we will see automatic, radio-like selection and broadcast of music content as a service in the near future, a real listener's community life also consists of enthusiastic radio moderators and a carefully selected mixture of music pieces, information and 
interaction. Both the majors and the radio stations have a lot to gain from "owning the customer". The following sections will discuss these potential issues.

\subsection{Challenges and opportunities for the radio station}

Data mining on aggregated buying patterns allows the radio stations to gain knowledge about their listeners which, in turn, could be immediately fed into their program design. For example, the buying behavior might be an excellent basis for pop music charts. Here we identify a complete feedback loop: Songs are in the charts, because many listeners ordered them and many further listeners can order the song, because it is frequently played on the radio. If there are some cheap buying options (not only: pay once- play forever, but also: pay per listen, pay per period etc.), there is a good chance that many will buy a song in order to contribute to the music charts and thus become customers for CYS. Information from CYS can also give feedback on the quality of program components and the program structure (e.g. the sequence of songs or: who prefers what kind of music when?).

Thus we consider that a distribution channel like CYS can have a significant impact on the value chain as indicated in figure 5 . We foresee a movement of radio stations into the selling, distribution and marketing element of the value chain, integrating primary and secondary exploitation. It remains an open question, whether they will also move into the publication and multiplication of audio media. Electronic distribution makes large sections of commercial multiplication of audio media obsolete; we rather see prosumers (Tapscott, 1996) not only consuming the audio media, but also configuring their own audio selection ${ }^{3}$.

If selling songs becomes a source of revenue for radio stations they might lose their innocence and credibility in the eyes of the listener; how much influence can or should cross-selling have on the design of the radio program? At one extreme, public radio stations will lose their special status if they become too commercialised. At the other extreme, private radio stations may become even more commercialised than they are today and other subjects of the music value chain may become interested in buying radio stations and using them as their distribution channel.

${ }^{3}$ It is a discussion beyond the scope of this paper, whether radio stations might also step up their efforts to publish, produce and sell their own content. As 'good' content is extremely expensive, we cannot yet imagine radio-stations participating in or even controlling the whole value chain. 


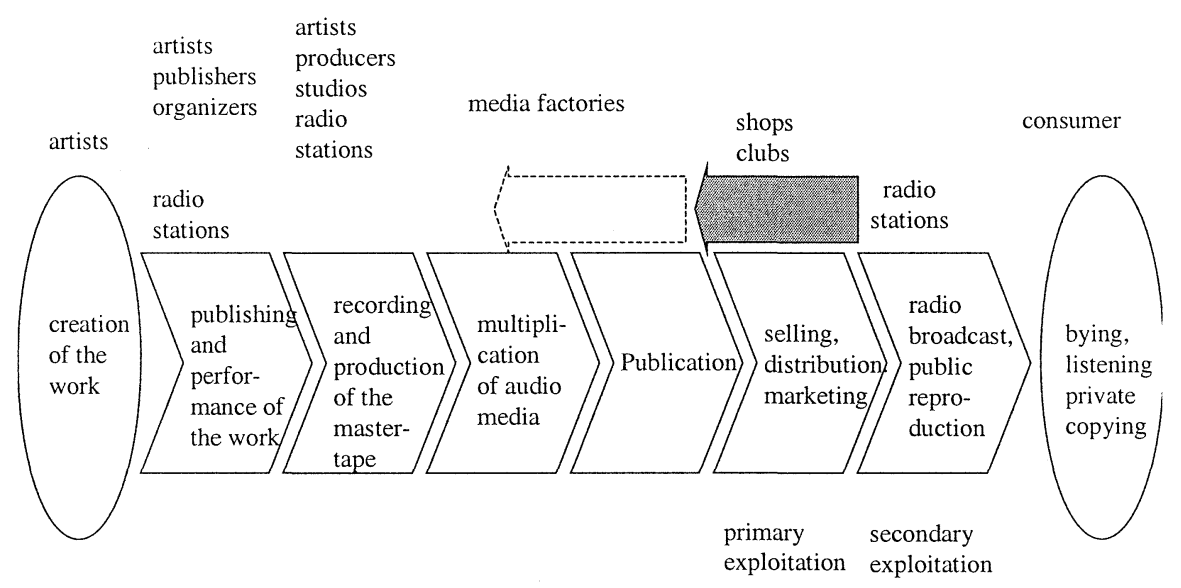

Figure 5. Radio stations' new role in the music industry value chain

\subsection{Challenges and opportunities for the content providers}

CYS can be more than an additional distribution channel for content providers - it can be the beginning of one-to-one marketing for music titles. As an additional channel it is only as valuable as its potential to generate additional revenue. Here it is an open question if CYS-buying would replace conventional purchasing or be on top of it. We would argue that instant buying has the potential to generate additional revenue because it originates from an emotional buying situation, but we do not yet have the empirical data to substantiate this assertion.

If the content provider persuade the radio stations to share the ownership of customer, or even leaves customer-ownership to the content providers, they can use the customer data for one-to-one marketing. This marketing then includes CYS, but can also be used for other channels, e.g. personalised follow-up-marketing ("If you like these two songs from this album, why don't you buy this album?"). Aggregated customer data can be used to 
configure and price new albums or other music selections. Allowing random configuration of titles might, however, lead to legal problems as current contracts frequently require the artist's consent to using his/her titles in other contexts.

\section{FURTHER RESEARCH QUESTIONS}

Although the legal problems might not been solved in the near future, most challenging would be to set up a test bed with a radio station and a mobile carrier. This could generate, over a limited period and for a closed user group, some empirical evidence concerning the acceptance and price sensitivity of this type of service. By starting with short audio clips instead of full length titles, the experimental study could be started even before the roll out of UMTS.

Later we see many more challenging research questions. A whole complex worthy of study are the interdependencies and stability of customer ownership scenarios.

From a more operational point of view it would be interesting to analyse the problems:

- If we assume we can receive superior media response data when counting visits to personalised web-pages and correlate them with buying transactions: how do we measure these data?

- What are the challenges of setting up sufficiently scalable and secure systems for mass traffic?

- Will the value chain be cut off by peer-to-peer approaches due to lower cost, as any new song can be digitally transferred without any loss of quality and with minimal delay by Gnutella-like systems? (Does this mean that even CYS will be too late?)

The general discussion on mobile commerce value chains mostly focuses on the later phases of the value chain. Figure 6 depicts such an understanding, and a similar example can be found in (Durlacher, 2001).

As can be observed, it summarises the value chain of the music industry in the "content provider" element. We have endeavoured to show that one element of the traditional value chain - the radio stations - have the potential to change the rules of the game. This leads to the question of whether those general models are really helpful in explaining the sophisticated mechanisms inherited from traditional domain-specific business-to-business interaction. Is it true that neither intermediaries nor carrier operators are found in pole 
position? Couldn't be the example presented here serve as a counterexample, if we consider re-intermediation by the radio and TV-stations?

We clearly see many more questions and problems, but our research so far has convinced us that mobile commerce will shift the opportunities and understanding of e-business into new dimensions.

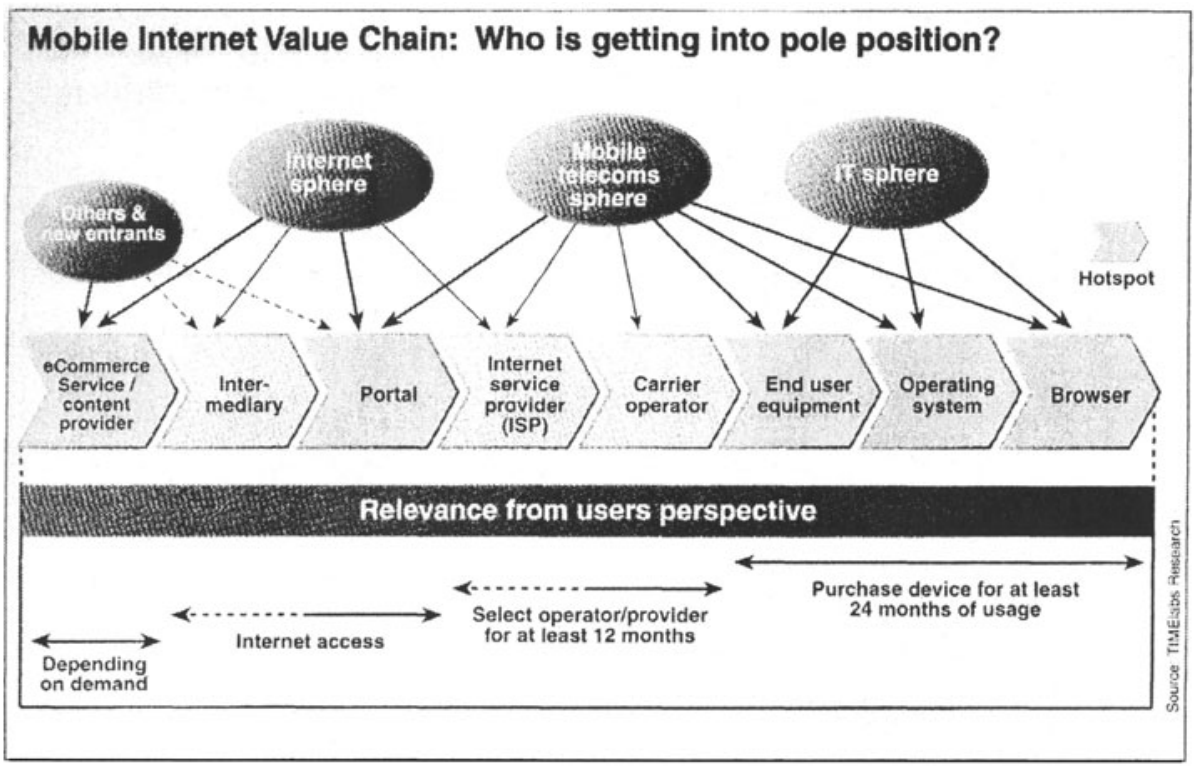

Figure 6. Generic mobile commerce value chain, taken from (TIMElabs 2000, p.36)

\section{ACKNOWLEDGEMENT}

We would also like to thank our Students Michael Biemer, Sascha Moschfeghi and Mustafa Oeren for the valuable input, particularily for implementing the CYS prototype.

\section{REFERENCES}

AMM (2001) www.iis.fhg.de/amm/ [Version: 6.2.2001]

AOD (2001), www.audio-on-demand.de/ [Version: 6.2.2001]

Dernbach (1998), Dernbach, B. et al. (ed.) (1998): Publizistik im vernetzten Zeitalter:

Berufe - Formen - Strukturen, Westdeutscher Verlag Opladen, Wiesbaden. 
Digitalradio (2001), www.digitalradio-info.de/ [Version: 6.2.2001]

Durlacher (2001), Durlacher Research. Mobile Commerce Report. www.durlacher.com/ research/res-reports.asp [Version: 6.2.2001]

Filk (1996), Filk, C. (1996): (Teil-)Dereguliertes Fernsehen: Eine kurze Rekapitulation. In: Friedrich-Ebert-Stiftung (ed.) (1996): Jahrbuch der Akademie der politischen Bildung 1996: Medien, Politik, politische Bildung, Friedrich-Ebert-Stiftung Bonn, S. 96-102.

Goldhammer/Zerdick (1999), Goldhammer, K.; Zerdick, A. (1999): Rundfunk Online: Entwicklung und Perspektiven des Internets für Hörfunk- und Fernsehanbieter, Vistas Verlag Berlin.

Gnutella (2001), gnutella.wego.com/ [Version: 6.2.2001]

Heinrich (1994), Heinrich, J. (1994): Medienökonomie, Band 1: Mediensystem, Zeitung, Zeitschrift, Anzeigenblatt, Westdeutscher Verlag Opladen 1994 (Reprint 2000).

Heinrich (1999), Heinrich, J. (1999): Medienökonomie, Band 2: Hörfunk und Fernsehen, Westdeutscher Verlag Opladen, Wiesbaden.

Heinzl et al. (2001), Heinzl, A.; König, W.; Hack, J.: Erkenntnisziele der Wirtschaftsinformatik in den nächsten drei und nächsten zehn Jahren. In: Wirtschaftsinformatik Vol 43, No. 3, p. 223.

IBM (2001) www.storage.ibm.com/hardsoft/diskdrdl/micro/datasheet.htm[Version: 6.2.2001] IIS, (2001), www.iis.fhg.de/amm/techinf/layer3/index.html[ Version: 6.2.2001] GmbH, Eschborn.

Kim (2000), Kim, A.: Community Building on the Web, Peachpit Press, Berkley.

Kulle (1998), Kulle, J.: Ökonomie der Musikindustrie, Peter Lang, Frankfurt et al.

Ludes/Werner (1997), Ludes, P.; Werner, A. (ed.) (1997): Multimedia-Kommunikation: Theorien, Trends und Praxis, Westdeutscher Verlag Opladen,Wiesbaden.

Marathe (2001), Marathe, J. (2001): Impacts of Digital Distribution on the Music Industry. Durlacher Research Report, http://www.durlacher.com/downloads/music.pdf [Version: 15.05.2002]

Middelhoff (2001), Middelhoff, T.: Auf in den digitalen Alltag der Musik!. Eröffnungsrede auf der Popkomm, 18. 8.2000, Köln. www.bertelsmann.de [Version: 6.2.2001]

MPEG (2001), www.mpeg.org/MPEG/aac.html [Version: 6.2.2001]

Mühl-Benninghaus/Zerdick (2000), Mühl-Benninghaus, W.; Zerdick, A. (ed.) (2000): Ökonomie der AV-Medien, Band 1: Fernsehen, Vistas Verlag Berlin.

Napster (2001), www.napster.com/ [Version: 6.2.2001]

Neverla (1998), Neverla, I. (ed.) (1998): Das Netz-Medium: Kommunikationswissenschaftliche Aspekte eines Medium in Entwicklung, Westdeutscher Verlag Opladen, Wiesbaden.

Nunamaker/Chen (1990), Nunamaker, Jr. J.F.; Chen, M.: Systems Development in Information Systems Research. In: Journal of Management Information Systems, Vol. 7. No. 3, 1990/91 p. 631-640.

Schumann (2000), Schumann, M.; Hess, T.: Grundfragen der Medienwirtschaft, Springer Berlin, Heidelberg.

Schwabe/Krcmar (2000), Schwabe, G.; Krcmar, H.: Piloting a Sociotechnical Innovation. In: Proceedings of the 8th European Conference on Information Systems ECIS 2000, Wirtschaftsuniversität Wien, Wien, S. 132-139.

SDMI (2001), www.sdmi.org [Version: 6.2.2001]SWR3 (2001), www.swr3.de/ [Version: $6.2 .2001]$ 
Seidel/Libertus (1993), Seidel N.; Libertus, M. (1993): Rundfunkökonomie: Organisation, Finanzierung und Management von Rundfunkunternehmen, Gabler Wiesbaden.

Siemens (2001) www.ic.siemens.com/mySiemens?product=SL45 [Version: 6.2.2001]

Szyperski (1971), Szyperski, N.: Zur wissensprogrammatischen und forschungsstrategischen Orientierung der Betriebswirtschaft. In: zfbf Vol 23 1971, S. 261-282.

Szyperski (1999), Szyperski, N. (ed.) (1999): Medienwirtschaft: Kompetenz - Akzeptanz Geschäftsfelder, Josef Eul Verlag Lohmar, Köln.

Tapscott (1996) Tapscott, D. (1996): The Digital Economy. Promise and Peril in the Age of Networked Intelligence. New York.

TIMElabs (2000), TIMElabs Research Center. Winning in Mobile eMarkets. Diebold Deutschland

Zerdick et al. (1999), Zerdick, A. et al.: Die Internet-Ökonomie - Strategien für die digitale Wirtschaft, Springer Berlin, Heidelberg. 